\section{Nível de entendimento de prescrições medicamentosas pediátricas em Unidades Básicas de Saúde}

\section{The level of understanding of pediatric prescription medicines at Basic Health Units}

Valeska de Oliveira Gonçalves Ferreira 1 Petr Melnikov 2

Mônica Cristina Toffoli-Kadri 3

\begin{abstract}
1,2 Programa de Pós Graduação em Saúde e Desenvolvimento da Região Centro Oeste. Departamento de Clínica Cirúrgica. Universidade Federal de Mato Grosso do Sul. Av. Senador Filinto Muller s.n. Campo Grande, MS, Brasil. CEP: 79.080-190. E-mail: valgferreira@hotmail.com

3 Departamento de Morfofisiologia. Universidade Federal de Mato Grosso do Sul. Campo Grande, MS, Brasil.
\end{abstract}

\section{Resumo}

Objetivos: avaliar o nivel de entendimento das prescrições medicamentosas pediátricas, pelos responsáveis das crianças, em Unidades Básicas de Saúde (UBS), municipio de Campo Grande, Mato Grosso do Sul.

Métodos: estudo transversal e descritivo, realizado entre maio de 2007 e junho de 2008. Foram entrevistados 336 acompanhantes de crianças, atendidas em UBS municipais, que receberam prescrição de medicamentos dispensados pela farmácia. Foram avaliados mediante questionário o nivel de escolaridade e fatores relacionados à compreensão da prescrição como legibilidade, nome, dose, freqüência de administração, tempo de uso de medicamentos, bem como a compreensão das informações verbais complementares.

Resultados: dos entrevistados, 80,4\% (270) eram mães, 55\%-(185) cursaram ensino fundamental e 90,2\% (303) receberam informações verbais complementares à prescrição. Das prescrições apresentadas $87,5 \%$ (294) foram consideradas legiveis. Dos entrevistados, 47\% (158) tiveram entendimento insuficiente da prescrição. $O$ entendimento insuficiente da prescrição foi associado à baixa escolaridade, ao não recebimento de informação verbal complementar e ao maior número de medicamentos presentes na prescrição.

Conclusões: é necessário que os responsáveis pela criança e profissionais envolvidos na prescrição e dispensação estejam conscientes da importância da informação sobre o medicamento, para a segurança das crianças.

Palavras-chave Pediatria, Prescrições de medicamentos, Compreensão 


\section{Introdução}

Diversos estudos têm evidenciado o uso não racional de medicamentos em crianças, especialmente em menores de dois anos. ${ }^{1-3}$ A Organização Mundial da Saúde (OMS) considera que estudos envolvendo esta população devem ser prioridade na área de pesquisa, porque, por motivos legais, éticos e econômicos, crianças não são incluídas em ensaios clínicos para desenvolvimento de novos medicamentos, sendo considerados assim órfãos terapêuticos. ${ }^{4}$

São inerentes ao paciente pediátrico algumas peculiaridades - como a imaturidade de seus sistemas responsáveis pela absorção, distribuição, metabolização e eliminação do medicamento - e a maior possibilidade de intoxicação por pequenas alterações de dose.5-7 Isto faz com que a compreensão da prescrição, por parte do responsável pela criança seja fator indispensável à segurança e eficácia da terapia proposta.

A responsabilidade da correta administração do medicamento à criança está intimamente ligada à essa compreensão, pois se o responsável não souber como usar o mesmo, é improvável que o faça adequadamente. ${ }^{7}$ Dentre os fatores que podem estar relacionados ao entendimento correto do uso do medicamento, idade e grau de escolaridade referemse ao responsável pela criança enquanto a legibilidade da prescrição, informações verbais complementares, tempo de consulta bem como a quantidade de medicamentos referem-se ao médico e ao profissional responsável por dispensar o medicamento na farmácia.7-10 Um bom entendimento da prescrição garante uma correta administração do medicamento e consequentemente maior sucesso no tratamento proposto, diminuindo o retorno ao sistema de saúde por ineficácia da terapia. 8

A comunicação adequada entre os profissionais de saúde e responsáveis pelas crianças, é indispensável para o sucesso do tratamento proposto e a prevenção de acidentes com medicamentos. Entretanto, ainda são poucas as referências que abordam o entendimento da prescrição pediátrica. Desta maneira o presente estudo avaliou o nível de entendimento das prescrições medicamentosas pediátricas, dos responsáveis pelas crianças, em Unidades Básicas de Saúde (UBS) em grande município do Estado do Mato Grosso do Sul.

\section{Métodos}

Esta é uma pesquisa transversal e descritiva que foi realizada no município de Campo Grande, Mato Grosso do Sul, o qual possui uma população esti- mada em aproximadamente 787.000 habitantes, de acordo com o censo demográfico 2010/IBGE (Instituto Brasileiro de Geografia e Estatística). ${ }^{11}$

O sistema de saúde local está organizado administrativamente em quatro distritos sanitários (Norte, Sul, Leste e Oeste) formados a partir do agrupamento das sete regiões urbanas que norteiam a administração municipal.

Fizeram parte do estudo os acompanhantes responsáveis pelas crianças atendidas nas 27 UBS que possuíam atendimento pediátrico e que obtiveram medicamentos na farmácia da unidade, no período de junho de 2007 a maio de 2008. É importante ressaltar que apesar de o farmacêutico estar presente em todas as farmácias das UBS estudadas, nenhuma delas possuía estrutura para atendimento farmacêutico individualizado. Os pacientes recebiam a medicação e orientações na fila, em pé, através de janelas presentes nas farmácias.

O cálculo do tamanho amostral foi baseado na média mensal de atendimentos pediátricos em cada UBS (320 atendimentos). Considerando uma margem de erro de cinco pontos percentuais, o tamanho da amostra foi de 368 indivíduos. Estes foram distribuídos nas UBS, totalizando 14 entrevistas em cada unidade.

O instrumento de coleta dos dados, previamente testado e validado, foi constituído por um questionário específico, com questões objetivas. As variáveis relacionadas aos acompanhantes foram: gênero, idade, escolaridade e grau de parentesco com a criança. As relacionadas à prescrição foram: o tipo de prescrição (manuscrita ou digitada), legibilidade, e fatores interferentes em seu entendimento como o nome do medicamento prescrito, indicação terapêutica, dose, via e frequência de administração, tempo de tratamento, identificação entre o medicamento obtido na farmácia e o prescrito e a compreensão da prescrição referida pelo entrevistado.

O nível de entendimento da prescrição foi classificado como insuficiente, mediano e suficiente e a metodologia utilizada foi adaptada de Sano et al., ${ }^{7}$ Ceccatto et al.,12 e Silva et al.13 O nível de compreensão para cada medicamento foi mensurado por meio de um escore, atribuindo-se diferentes pontos para cada item, de acordo com sua importância para o uso seguro do medicamento. Para informações como nome do medicamento e indicação terapêutica foi atribuído um ponto, enquanto para dose, via de administração, frequência de administração, tempo de tratamento e identificação positiva do medicamento prescrito com o obtido na farmácia foram atribuídos três pontos.

O total da pontuação poderia assim atingir até 17 
pontos. Desta forma o entendimento foi considerado suficiente para cada medicamento quando o escore atingia 17 ou 16 pontos; mediano com 15 pontos e insuficiente entre 14 e 0 pontos. O corte em 14 pontos para classificação em entendimento insuficiente, considerou que o responsável pela criança não poderia apresentar falha de compreensão nos parâmetros considerados imprescindíveis (três pontos) à segurança do paciente pediátrico.

Para a avaliação do entendimento da prescrição completa, com vários medicamentos, foi necessário desenvolver uma metodologia apropriada, adaptada de Likert. ${ }^{14}$ Para tanto, foram atribuídos valores numéricos simétricos relacionados aos diferentes níveis de entendimento para cada medicamento, como indicado na Figura 1.

Para a variável legibilidade, foi considerado legível o medicamento lido sem qualquer dúvida; ilegível aquele em que foi impossível a leitura e parcialmente legível quando lido em partes, mas não na totalidade. Utilizando a mesma escala de valores simétricos citada anteriormente, para cada medicamento da prescrição foi atribuído um valor, de acordo com a classificação proposta, sendo -1 para ilegível, 0 para parcialmente legível e +1 para legível. A legibilidade da prescrição como um todo foi definida pela somatória das atribuições classificadas para cada medicamento da prescrição.

Os dados foram analisadas pelo teste quiquadrado de Pearson. As análises estatísticas foram feitas usando os programas SPSS versão 10.0 e 16.0 para Windows.

O trabalho foi aprovado pelo Comitê de Ética e Pesquisa da Universidade Federal de Mato Grosso do Sul (UFMS) sob o protocolo 934/2007.

\section{Resultados}

Durante a coleta de dados, três das vinte e sete UBS deixaram de contar com o serviço de pediatria, inviabilizando 32 entrevistas. Nas 24 UBS restantes foram totalizadas 336 entrevistas.

A caracterização dos acompanhantes responsáveis pelas crianças atendidas nas UBS de Campo Grande teve 92\% (311) de mulheres, com ensino fundamental declarado 55\% (185). Destas, $80,4 \%$ (270) eram mães das crianças atendidas. A média de idade foi de $28 \pm 9,67$ anos, variando entre 17 e 64 anos.

Das prescrições analisadas, 334 foram manuscritas, uma foi apresentada na forma de carimbo padronizado e uma digitada. O número médio de medicamentos por prescrição foi $1,9 \pm 0,9$, variando entre 1 e 5 . Não houve nenhuma prescrição considerada ilegível segundo os critérios adotados na metodologia (Tabela 1).

Ao todo foram prescritos 644 medicamentos nas 336 prescrições. Para cada uma dessas, foi avaliado se o responsável pela criança havia compreendido as seguintes informações: nome, dose, via e frequência de administração, indicação terapêutica, tempo de tratamento de cada medicamento da prescrição e

Figura 1

Classificação do entendimento da prescrição pediátrica em Unidades Básicas de Saúde do município de Campo Grande, MS, 2008.

\begin{tabular}{lc}
\hline Item avaliado & Pontuação atribuída por item \\
\hline Nome do medicamento & 1 Ponto \\
Indicação terapêutica & 1 Ponto \\
Dose & 3 Pontos \\
Via de administração & 3 Pontos \\
Frequência administração & 3 Pontos \\
Tempo tratamento & 3 Pontos \\
Identificação positiva do medicamento & 3 Pontos
\end{tabular}

\begin{tabular}{|c|c|c|c|c|}
\hline \multicolumn{3}{|c|}{ Nível de entendimento por medicamento } & \multicolumn{2}{|c|}{ Nível de entendimento da prescrição } \\
\hline $\begin{array}{l}\text { Somatória da pontuação } \\
\text { dos Itens }\end{array}$ & Classificação atribuída & Score atribuído & Pontuação atingida & Classificação atribuída \\
\hline 17 ou 16 pontos & Suficiente & +1 & $\geq+1$ & Suficiente \\
\hline 15 pontos & Mediano & 0 & 0 & Mediano \\
\hline 0 a 14 pontos & Insuficiente & -1 & $\leq-1$ & Insuficiente \\
\hline
\end{tabular}


capacidade de identificar o medicamento dispensado na farmácia com o descrito na prescrição (Tabela 2).

Quando questionados sobre o recebimento de informações verbais complementares à prescrição, $90,2 \%$ (303) dos entrevistados afirmaram ter recebido informações adicionais durante a consulta e/ou dispensação. Os médicos foram citados como os únicos responsáveis por esta informação verbal complementar por 48,2\% (163) dos entrevistados, médicos e farmacêuticos por $27,1 \%$ (91) e farmacêutico isoladamente foi citado como único responsável por $14 \%$ (47). Enfermeiros foram lembrados em 1\% (2) das entrevistas.

$\mathrm{Na}$ avaliação do entendimento da prescrição, segundo critérios adotados na metodologia, 43,5\% (146) dos entrevistados demonstraram entendimento suficiente, 47\% (158) insuficiente e 9,5\% (32) mediano. No entanto, os responsáveis entrevistados, relataram uma percepção de entendimento distinta da definida na pesquisa, pois $84,2 \%$ (283) dos entrevistados afirmaram ter compreendido totalmente a receita e as informações pertinentes à administração do medicamento à criança. Vale ressaltar que $22,9 \%$ (77) deles não eram o responsável direto pela administração do medicamento à criança, ou seja, teriam que explicar a terceiros como fazê-lo.

Foi realizada a análise do entendimento da prescrição de acordo com os níveis de escolaridade (analfabeto ou ensino fundamental) e (ensino médio ou superior) versus os níveis de entendimento (insuficiente, mediano e suficiente) como pode ser visto na Tabela 3.

Tabela 1

Características das prescrições pediátricas de acordo com legibilidade, tipo e número de medicamentos, em Unidades Básicas de Saúde do município de Campo Grande, MS, 2008 ( $n=336)$.

\begin{tabular}{lcc}
\hline Características & N & $\%$ \\
\hline Tipo de prescrição & 334 & 99,4 \\
Manuscrita & 01 & 0,3 \\
Digitada & 01 & 0,3 \\
Carimbo & & 37,2 \\
Medicamentos por prescrição & 125 & 41,1 \\
1 & 138 & 17,3 \\
2 & 58 & 3,3 \\
3 & 11 & 1,2 \\
4 & 04 & 87,5 \\
5 & & 12,5 \\
Llassificação de legibilidade* & 294 & 0,0 \\
Parcialmente legível & 42 & 0 \\
Ilegível & 04 & \\
\hline
\end{tabular}

*Legível= prescrição lida sem dúvida; Ilegível= impossível a leitura da prescrição; Parcialmente legível= prescrição lida em partes, mas não na totalidade.

Tabela 2

Compreensão dos parâmetros contidos nas prescrições pediátricas de medicamentos em Unidades Básicas de Saúde do município de Campo Grande, MS, 2008 ( $n=644)$.

\begin{tabular}{lll}
\hline Parâmetros & \multicolumn{2}{c}{ Compreensão positiva } \\
\cline { 2 - 3 } & $\mathrm{n}$ & $\%$ \\
\hline & & 76,7 \\
Nome & 494 & 68,8 \\
Dose & 443 & 86,8 \\
Via de administração & 559 & 80,6 \\
Frequência de administração & 519 & 83,1 \\
Indicação terapêutica & 535 & 69,7 \\
Tempo de tratamento & 449 & 95,2 \\
Identificação entre medicamento prescrito e dispensado & 613 &
\end{tabular}


Houve associação entre ser analfabeto ou ter cursado ensino fundamental e apresentar entendimento insuficiente e ter cursado ensino médio ou superior e apresentar entendimento suficiente $(p<0,001)$.

$\mathrm{O}$ recebimento de informações verbais complementares mostrou-se associado a um melhor nível de entendimento das prescrições $(p<0,024)$ (Tabela 4$)$.
Também foi possível observar uma associação entre a polifarmacoterapia e o entendimento das prescrições. Prescrições contendo apenas um medicamento foram classificadas como de entendimento insuficiente por $52,8 \%(66 / 125)$ dos responsáveis, enquanto prescrições contendo cinco medicamentos foram classificadas como entendimento insuficiente em $100 \%(4 / 4)$ dos casos $(p=0,01)$.

\section{Tabela 3}

Associação entre o nível de entendimento das prescrições pediátricas e o grau de escolaridade dos entrevistados em Unidades Básicas de Saúde do município de Campo Grande, MS, 2008 ( $n=336)$.

\begin{tabular}{|c|c|c|c|c|c|c|c|}
\hline \multirow[t]{3}{*}{ Escolaridade } & \multicolumn{6}{|c|}{ Nível de entendimento } & \multirow[t]{3}{*}{$p^{*}$} \\
\hline & \multicolumn{2}{|c|}{ Insuficiente } & \multicolumn{2}{|c|}{ Mediano } & \multicolumn{2}{|c|}{ Suficiente } & \\
\hline & $\mathrm{n}$ & $\%$ & $\mathrm{n}$ & $\%$ & $\mathrm{n}$ & $\%$ & \\
\hline Analfabeto ou ensino fundamental & 102 & 55,1 & 21 & 11,4 & 62 & 33,5 & $<0,001$ \\
\hline Ensino médio ou superior & 56 & 37,1 & 11 & 7,3 & 84 & 55,7 & \\
\hline Total & 158 & 47,0 & 32 & 9,5 & 146 & 43,5 & - \\
\hline
\end{tabular}

${ }^{*} \chi^{2}$ de Pearson=16,6; $\mathrm{gl}=2$

Tabela 4

Associação entre o nível de entendimento das prescrições pediátricas e o recebimento de informação verbal complementar em Unidades Básicas de Saúde do município de Campo Grande, MS, 2008 (n=336).

\begin{tabular}{|c|c|c|c|c|c|c|c|}
\hline \multirow[t]{3}{*}{ Recebimento de informação verbal } & \multicolumn{6}{|c|}{ Nível de entendimento } & \multirow[t]{3}{*}{$p^{*}$} \\
\hline & \multicolumn{2}{|c|}{ Insuficiente } & \multicolumn{2}{|c|}{ Mediano } & \multicolumn{2}{|c|}{ Suficiente } & \\
\hline & $\mathrm{n}$ & $\%$ & $\mathrm{n}$ & $\%$ & $\mathrm{n}$ & $\%$ & \\
\hline Não & 23 & 69,7 & 2 & 6,1 & 8 & 24,2 & 0,02 \\
\hline Sim & 135 & 44,6 & 30 & 9,9 & 138 & 45,5 & \\
\hline Total & 158 & 47,0 & 32 & 9,5 & 46 & 43,5 & - \\
\hline
\end{tabular}

${ }^{*} \chi^{2}$ de Pearson=7,57; $\mathrm{gl}=2$

\section{Discussão}

Embora a maioria dos acompanhantes das crianças fossem mães, jovens e com escolaridade satisfatória para capacidade de leitura, o não entendimento da prescrição por boa parte delas, mostra que este não é o único fator relacionado à compreensão. No Brasil, $9,9 \%$ da população é analfabeta e $59 \%$ dos alunos com até quatro anos de ensino são enquadrados como analfabetos funcionais. $15 \mathrm{Em}$ Mato Grosso do Sul a média de escolaridade encontra-se em 7,2 anos, ou seja, ensino fundamental.11

Verificou-se também maior compreensão da prescrição por quem possuía maior escolaridade. Estudos sobre nível de entendimento e informação de prescrições indicam a escolaridade, o que suporta dados anteriores indicando a escolaridade como um dos fatores determinantes para a compreensão das prescrições. $7,13,16-19$

Dois outros estudos realizados em Santa Catarina obtiveram resultados semelhantes a esta pesquisa. Leite e Vasconcelos 20 identificaram que o gênero feminino com idade entre 21 e 40 anos e ensino fundamental predominou entre os responsáveis pelas crianças. Carvalho et al. ${ }^{21}$ mostraram que $75 \%$ dos entrevistados eram mães com oito anos de estudo em média. Também Sano et al.7 avaliaram o entendimento das prescrições pediátricas $\mathrm{e}$ constataram que $84 \%$ dos acompanhantes eram mães de crianças atendidas e $63 \%$ dessas possuíam o ensino fundamental incompleto, encontrando associação entre maior escolaridade e melhor compreensão das prescrições. 
$\mathrm{O}$ número médio de medicamentos por prescrição aqui verificado está de acordo com o preconizado pela Organização Pan Americana de Saúde e Organização Mundial da Saúde, 22 que é de 1,3 a 2,2 medicamentos sendo que, menos de um quarto das prescrições continha três ou mais medicamentos. Este é um resultado positivo, quando consideramos a vulnerabilidade das crianças frente a vários medicamentos administrados concomitantemente. Pesquisas semelhantes mostraram resultados distintos, onde a polimedicação foi predominante nas prescrições pediátricas, ocasionando o aparecimento de reações adversas a medicamentos (RAMs) e intoxicações. ${ }^{2,23}$

No presente estudo foi observado que a maior quantidade de medicamentos prescritos esteve também associada ao menor entendimento da prescrição. Como um maior volume de informações na prescrição exige maior detalhamento nas orientações verbais tanto durante a consulta quanto na dispensação, a informação contida na prescrição muitas vezes é insuficiente para a compreensão dos responsáveis. Outros autores 7,9,12,13,24 apontam perfil semelhante, mostrando a insuficiência do entendimento associada ao maior número de medicamentos prescritos.

Habitualmente, a presença de abreviaturas ou símbolos, assim como a ilegibilidade, dificultam o entendimento de prescrições. 7,25,26 Neste trabalho, no entanto, $87,5 \%$ (294) das prescrições estavam legíveis e a presença das abreviaturas não foram associadas ao não entendimento.

Quando considerada a dose e o tempo de tratamento, foi aqui observado que aproximadamente um terço dos medicamentos prescritos não teve estes parâmetros compreendidos. Resultados semelhantes foram obtidos por Silva et al., $13 \mathrm{em}$ que um quinto dos pacientes entrevistados não sabia a dose do medicamento a ser usado e aproximadamente um terço desconhecia o tempo de tratamento e não sabia informar a frequência de administração. Em trabalho semelhante, Sano et al. ${ }^{7}$ mostraram que parte dos acompanhantes das crianças também não compreendiam a posologia dos medicamentos prescritos. A incompreensão do todo ou parte da prescrição pode originar erros de medicação, que, graves ou não, comprometem a eficácia do tratamento e podem colocar em risco a vida da criança que está sendo medicada. A participação da família nos cuidados à criança deve, portanto abranger inclusive a consciência da administração correta do medicamento.

$\mathrm{Na}$ presente pesquisa, as prescrições apresentaram apenas a posologia como orientação escrita, contudo a orientação verbal complementar à prescrição foi feita em $90,2 \%$ dos atendimentos. Apesar disso, o risco da má administração do medicamento, por aqueles que não tiveram orientação dos profissionais da saúde, não está descartado.

Contrariamente, a literatura revela situações preocupantes relacionadas à falta de orientação verbal complementar à prescrição. 24,27

Evidências da literatura mostram uma relação direta entre o bom nível de entendimento das prescrições e a existência de orientação verbal da equipe de saúde.12,28 Como a produção e transmissão de informações não se traduzem necessariamente em conhecimento é necessário que tanto o médico como o farmacêutico estejam disponíveis para adequar a linguagem utilizada ao entendimento dos responsáveis pela criança. 28

A diferença acentuada entre indivíduos que afirmavam ter compreendido completamente a receita, mas não serem classificados como tal, é uma contradição perigosa, pois se os responsáveis acreditam saber o necessário, podem não solicitar maiores esclarecimentos ou menosprezar informações fornecidas durante a consulta e dispensação do medicamento. Assim todo o tratamento poderá estar comprometido, seja em relação à adesão, eficácia ou aos riscos inerentes em decorrência da falta de preparo dos responsáveis pelo tratamento. Sano et al., 7 encontraram uma diferença de $10 \%$ entre o nível de compreensão da prescrição aferido em sua pesquisa e o informado pelos entrevistados.

Os profissionais mais citados como responsáveis pela explicação adicional à prescrição foram os médicos, seguidos pelos farmacêuticos. No entanto, outros estudos demonstraram que os profissionais farmacêuticos são os mais lembrados quanto às informações prestadas sobre a utilização dos medicamentos. 9,12

Como o número de entrevistados com nível insuficiente de entendimento da prescrição foi expressivo, isto pode significar um risco em potencial à segurança dos pacientes pediátricos, que sequer são responsáveis por sua própria terapia. $\mathrm{O}$ pequeno número de estudos semelhantes 7,16,19 a este, avaliando o entendimento de prescrições pediátricas é surpreendente, considerando que crianças são pacientes sensíveis à presença de fármacos em seu organismo e susceptíveis a reações adversas.

A assistência primária à saúde sendo falha, acaba por sobrecarregar os demais níveis institucionais da saúde pública, aumentando os custos, sendo boa parte destes com medicamentos. 29 Neste sentido, é importante propor que os tradicionais estudos de 
utilização de medicamentos sejam complementados por pesquisas que abordem questões qualitativas relacionadas a este uso, ressaltando a condição das prescrições e da comunicação entre a equipe de saúde e os usuários dos serviços de pediatria nas UBS.

\section{Considerações finais}

É importante que médicos e farmacêuticos estejam atentos às dificuldades e limitações da compreensão da prescrição, para que seja garantida a segurança da criança. A adequação da linguagem da equipe de saúde e a certificação do entendimento das orientações prestadas são fatores imprescindíveis para a garantia de sucesso. Falhas de compreensão acarretarão falhas na terapia.

Sugestões para proporcionar um melhor entendimento das prescrições pediátricas podem ser formuladas a partir dos dados gerados nesta pesquisa, como a elaboração de um programa piloto de atenção farmacêutica para pacientes pediátricos, com esquema de dispensação individualizada e

\section{Referências}

1. Béria JU, Victora CG, Barros FC, Teixeira AB. Lombardi C. Epidemiologia do consumo de medicamentos em crianças de centro urbano da região sul do Brasil. Rev Saúde Pública. 1993; 27: 95-104.

2. Bricks LF, Leone C. Utilização de medicamentos por crianças atendidas em creches. Rev Saúde Pública. 1996; 30: $527-35$.

3. Bricks LF. Uso judicioso de medicamentos em crianças. J Pediatr (Rio J). 2003; 79. Supl.1:107-14.

4. WHO (World Health Organization). The use of essential drugs: Seven report of the WHO Expert Committee. Technical report series, n. 867. Geneva; 2000.

5. Alcântara DA, Vieira LJES, Albuquerque VLM Intoxicação medicamentosa em criança. Rev Bras Promoção Saúde. 2003; 16: 10-6.

6. Alcorn J, MacNamara PJ. Pharmacokinetics in the newborn. Adv Drug Deliv Rev. 2003; 55: 667-86.

7. Sano IP, Masotti RR, Santos AAC, Cordeiro JA. Avaliação do nível de compreensão da prescrição pediátrica. J Pediatr (Rio J). 2002; 78: 140-5.

8. Chemello C, Castro MS. Adaptação de método de orientação de pacientes sobre medicamentos por uma análise de compreensão. Acta Farm Bonaer. 2006; 25: 613-8.

9. Cunha MCN, Zorzatto JR, Castro LLC. Avaliação do uso de medicamentos na rede pública municipal de saúde de Campo Grande/MS. Rev Bras Ciênc Farm. 2002; 38: 21527.

10. Monreal MTFD, Cunha RV, Trinca LA. Compliance and retroviral medication as reported by AIDS patients assisted acompanhamento farmacoterapêutico. Cursos de capacitação para a equipe de saúde, que abordem as questões relacionadas à qualidade da comunicação entre profissionais e pacientes, também podem ser implantados. Podem ainda ser oferecidas palestras de cunho educativo aos usuários dos serviços de pediatria, para a conscientização da necessidade do comprometimento da família quanto à segurança no uso de medicamentos em crianças.

\section{Agradecimentos}

À Secretaria Municipal de Saúde de Campo Grande, Mato Grosso do Sul, pela oportunidade da realização deste trabalho.

À Profa. Dra. Maria Beatriz Cardoso Ferreira (UFRGS), pela valiosa colaboração, nas correções e observações ao artigo.

Ao Prof. Dr. Celso Corrêa (UNIDERP) e à Farmacêutica Úrsula Jacobs (UFRGS), pela colaboração na análise estatística. at the University Hospital of the Federal University of Mato Grosso do Sul. Braz J Inf Dis. 2002; 6: 8-14.

11. IBGE (Instituto Brasileiro de Geografia e Estatística) Indicadores culturais; 2010.

12. Ceccato MGB, Acurcio FA, Bonolo PF, Rocha GM, Guimarães MDC. Compreensão de informações relativas ao tratamento anti-retroviral entre indivíduos infectados pelo HIV. Cad Saúde Pública. 2004; 20: 1388-97.

13. Silva T, Schenckel EP, Mengue SS. Nível de informação a respeito de medicamentos prescritos a pacientes ambulatoriais de hospital universitário. Cad Saúde Pública. 2000; 16 : 449-55.

14. Likert R. A technique for the measurement of attitudes. Arch Psychol. 1932; 22: 1-55.

15. INEP (Instituto Nacional de Estudos e Pesquisas Educacionais Anísio Teixeira). Manual do Analfabetismo no Brasil; 2001.

16. Arnhold RG, Adebonojo FO, Callas ER, Callas J, Carte E, Stein RC. Patients and prescription: comprehension and compliance with medical instruction in a suburban pediatric practice. Clin Pediatr. 1970; 9: 648-51.

17. Wolf MS, Davis TC, Tilson HH, Bass PF, Parker RM. Misunderstanding of prescriptions drug warning labels among patients with low literacy. Am J Health-System Pharm. 2006; 63: 1048-55.

18. Davis TC, Wolf MC, Bass PF, Thompson JA, Tilson HH, Neuberger M, Parker RM. Literacy and misunderstanding prescriptions drug labels. Ann Int Med. 2006; 145: 887-94. 
19. Menezes APS, Domingues MR, Baisch ALM Compreensão das prescrições pediátricas de antimicrobianos em unidades de saúde em um município do sul do Brasil. Rev Bras Epidemiol. 2009; 12: 478-89.

20. Leite SN, Vasconcelos MPC. Adesão à terapêutica medicamentosa: elementos para discussão de conceitos e pressupostos adotados na literatura. Ciênc Saúde Coletiva. 2003; 8: 775-82.

21. Carvalho DC, Trevisol FS, Menegali BT, Trevisol DJ. Uso de medicamentos em crianças de zero a seis anos matriculadas em creches de Tubarão, Santa Catarina. Rev Paul Pediatr. 2008; 26: 238-44.

22. OPAS/OMS (Organização Pan Americana de Saúde/Organização Mundial da Saúde). Série medicamentos essenciales y tecnologia. Guia para el desarrollo de servicio farmacêutico hospitalario e atención farmaceutica al paciente ambulatório. Washington, DC; 1997.

23. Santos DB, Coelho HLL. Reações adversas a medicamentos em pediatria: uma revisão sistemática de estudos prospectivos. Rev Bras Saúde Matern Infant. 2004; 4: 341 -

24. Arrais PSD, Barreto ML, Coelho HLL. Aspectos dos processos de prescrição e dispensação de medicamentos na percepção do paciente: estudo de base populacional em Fortaleza, Ceará, Brasil. Cad Saúde Pública. 2007; 23: $927-$ 37.

Recebido em 4 de março de 2011

Versão final apresentada em 8 de junho de 2011

Aprovado em 11 de julho de 2011
25. Naves JOS, Silver LD. Evaluation of pharmaceutical assistance in public primary care in Brasília, Brazil. Rev Saúde Pública. 2005; 39: 223-30.

26. Aguiar G, Silva Jr LA, Ferreira MAM. Ilegibilidade e ausência de informação nas prescrições médicas: fatores de risco associados à erros de medicação. Rev Bras Promoção Saúde. 2006; 19: 84-91.

27. Badia LX, Magaz MS, Gutierrez NL, Guilera SM. Prescription medicine information: Spanish general population survey. Aten Primaria. 2005; 36: 93-9.

28. Pepe VLE, Osório-de-Castro CGS. A interação entre prescritores, dispensadores e pacientes: informação compartilhada como possível benefício terapêutico. Cad Saúde Pública. 2000; 16: 815-22.

29. Osório-de-Castro CGS. Estudos de utilização de medicamentos: noções básicas. Rio de Janeiro: Fiocruz; 2002. 